Traditional Medicine and Modern Medicine

Vol. 2, No. 2 (2019) 31-41

(C) Institutes of Integrative Medicine, Fudan University and

World Century Publishing Corporation

DOI: $10.1142 /$ S2575900019100013

\title{
Origin and future of medicine
}

\author{
Wenxian Liu ${ }^{1,3}$, Zhen Gao ${ }^{1,3}$, Linwei Lu $^{2,3}$, Hu Zhang ${ }^{3,4}$, Shan Jiang ${ }^{3,4}$, \\ Cheng $\mathrm{Ma}^{3,4}$, Zhao Tang ${ }^{3,4}$, Qiuping $\mathrm{Li}^{3,4}$, Nabijan Mohammadtursun ${ }^{3,4}$, Lingli $\mathrm{Hu}^{3,4}$, \\ Wuniqiemu Tulake ${ }^{3,4}$, Zexi $\mathrm{Lv}^{3,4}$ and Jingcheng Dong ${ }^{2,3, *}$ \\ ${ }^{1}$ The Postdoctoral Research Station of Integrative Medicine \\ Fudan University, 220 Handan Road \\ Shanghai 200433, P. R. China \\ ${ }^{2}$ Department of Integrative Medicine, Huashan Hospital \\ Fudan University, 12 Middle Urumqi Road \\ Shanghai 200040, P. R. China \\ ${ }^{3}$ Institute of Theories and Application \\ Institutes of Integrative Medicine, Fudan University \\ 12 Middle Urumqi Road, Shanghai 200040, P. R. China \\ ${ }^{4}$ The Graduate School, Fudan University \\ 220 Handan Road, Shanghai 200433, P. R. China \\ *jcdong2004@126.com
}

Received 15 May 2019; Accepted 30 May 2019; Published 8 July 2019

\begin{abstract}
Based on the comprehensive observation of medicine, we made a diachronic summarization and synchronic comparison on medicine, including traditional medicine and modern medicine as well as Chinese traditional medicine (CTM) and Western traditional medicine. We focused on the connotation of several predominant medicines, the evolution of medical civilization, the problems and challenges of medicine at present, the prospect and framework of medicine in the future, and finally put forward the new system of CTM together with the integration of traditional medicine and modern medicine so as to contribute to the thinking, research and construction of medicine for mankind, which will add to its positive development and scientific practice.
\end{abstract}

Keywords: Medicine; traditional medicine; modern medicine; Chinese traditional medicine (CTM).

\section{Introduction}

Medicine, including traditional medicine and modern medicine, has kept developing for a long time. Tremendous progress has been made, and yet new problems and challenges are continuously emerging. At this new historic beginning, it remains an important topic worthy of thinking and practicing for every medical practitioner and even for the general public what the history and future of medicine is (especially traditional medicine), and it is also a critical issue with theoretical and practical significance.

\section{The Definition of Medicine}

It seems that everyone is familiar with medicine, but it is not so easy to define it scientifically. The connotation and essence of medicine keep evolving with the progression of

\footnotetext{
*Corresponding author.
} 
human civilization and the advance of medical science, and the understanding of medicine and its ways of understanding vary from different cultures.

What is Medicine? Ci Hai (an unabridged and comprehensive dictionary of Chinese) defines it as "a scientific system to study the process of human life and the struggle against diseases". ${ }^{1}$ The Contemporary Chinese Dictionary gives a definition that medicine is "the science of protecting and promoting human health, preventing and treating diseases". ${ }^{2}$ Wikipedia refers that "medicine is the science and practice of diagnosis, treatment and prevention of diseases". ${ }^{3}$ In conclusion, medicine is the science of diagnosis, treatment, and prevention of diseases by studying the process of human life so as to promote human health.

What is Modern Medicine? People's understanding of the concepts and ways of understanding are complex and diverse, which is determined by different cultural backgrounds and different development of medicine. Generally, at present, there are only medicine and complementary/alternative medicine in most countries around the world, and the predominant medicine is modern medicine. However, in China, modern medicine usually refers to "Western medical system since the modern times", and the science and practice of preventing and treating diseases based on such disciplines as anatomy, physiology, histology and embryology, biochemistry and molecular biology, pathology, etc. Though originating from Western countries, modern medicine has become predominant in the world.

What is Traditional Medicine? According to World Health Organization (WHO), traditional medicine refers to the knowledge, skills and practice based on the theories, beliefs and experiences peculiar to different cultures, which is used in the maintenance of health and in the prevention, diagnosis, improvement or treatment of physical and mental diseases. Traditional medicine varies in different countries, regions and civilizations, leading to the differences in the treatment and practice. It is also called "Alternative medicine" or "Complementary medicine" in some countries. ${ }^{4}$

What is Chinese traditional medicine (CTM)? CTM, also called traditional Chinese medicine (TCM, Zhong Yi), is a concept that is constantly changing, deepening and enriching with the evolution of the history of traditional medicine in China, and the development of medical practice. From the disciplinary perspective, it has evolved from a primitive regional medical concept to a hierarchical comprehensive one, and then turned into the counterpart of Western medicine/ modern medicine to some extent. It has also evolved from the concept of Han Medicine to that of traditional medicines including Han and other minority medicines. ${ }^{5}$ In 2014, we published the articles that proposed the concepts of CTM ( $D a$ Zhong Yi), Trichotomy (San Fen Fa) and Five Key Elements $(\mathrm{Wu} \text { Yao Su})^{6,7}$ for the reconstruction of TCM in the mainstream media such as People's Daily. CTM (Da Zhong $Y i)$ is the general appellation of all the traditional medicines of different ethnicities in China, including Han Medicine, Tibetan Medicine, Mongolian Medicine, Uyghur Medicine, Dai Medicine, Zhuang Medicine, Miao Medicine, Yao Medicine, Hui Medicine, etc. Five Key Elements (Wu Yao Su) refer to clinical experience, ancient philosophy, regional culture, religious belief, and primitive knowledge of medicine, which integratively frame any traditional medicine. Trichotomy (San Fen Fa) is aimed at the overall construction of CTM and is conducive to understanding and distinguishing the relationship between CTM and modern medicine. We propose that the basic structure of any traditional medicine can be divided into the following three parts: the part that is unconsciously ahead of modern medicine, the part that has already reached consensus with modern medicine, and the part that needs to be reconsidered or abandoned. In the Law of the People's Republic of China on Traditional Chinese Medicine implemented in 2017, it is clearly stated in the "General Provisions" that "TCM is the general term of medicine of all Chinese ethnic groups including the Han ethnic group and minority ethnic groups, reflects the understanding of the Chinese nation on life, health and diseases, and is a medicine and pharmacology system with long historical traditions and unique theories and technical methods."

What is Integrative Medicine? In 1956, Chairman Mao proposed that "We should combine the knowledge of TCM with that of Western medicine so as to create a unified new medicine in China." 8 The concept of integrative medicine in academia is derived from this statement, and it has not only laid the foundation for the discipline of integrative medicine, but also emphasized the innovative significance of integrative medicine and its importance in the disciplines of medicine in China. From the perspective of the development of medicine in the world, integrative medicine is quite precious in the background that a majority of countries have preferred modern medicine to traditional medicine since modern times. Simultaneously, integrative medicine has become increasingly attractive since most countries have gradually recognized the limitations of modern medicine and reconsidered traditional medicine. Therefore, integrative medicine, the integration of traditional medicine and modern medicine, has become a medical discipline that might represent the direction of medical development in the future. Created in China though, integrative medicine also belongs to the world.

Medicine should be universal and international. Whether initially or ultimately, medicine is the science of diagnosis, treatment and prevention of diseases so as to promote human health. However, considering the current development of medicine, different cultural backgrounds and different understandings of medicine in different countries and nationalities, today there are still some medical conceptual 
distinctions in the world, including modern medicine versus traditional medicine, predominant medicine versus complementary/alternative medicine, TCM versus Western medicine, etc. For example, traditional medicine is composed of Western traditional medicine and Eastern traditional medicine, which, of course, includes TCM that has been representing the high level of traditional medicine. After the Renaissance, modern medicine, a medical system rooted in Western traditional medicine, was gradually established thanks to the Industrial Revolution as well as the Scientific and Technological Revolution, keeping predominant in contemporary medicine. Predominant medicine plus complementary/alternative medicine is a new medical pattern in the West while TCM plus Western medicine is a common medical pattern in China after the Opium War.

\section{The Brief History of Medicine}

When did medicine originate? It is generally recognized that medicine come along with human beings. The famous medical historian Arturo Castiglioni said that "medicine was born with the first expression of suffering and the first desire to alleviate this suffering". ${ }^{9}$ However, in the strict sense, medicine or even medical skills did not appear in the primitive hunting and farming period. The simple behaviors, such as warming themselves with fire, draping themselves with animal furs, repelling insects with mud, might be their defense by instinct.

With the development of human civilization, for example, simple written words appeared on the basis of the primitive human languages, cities were established after the period of group living and settlement, and then medical civilization, an important component of human civilization, began to display to the world in a presentable way. The Code of Hammurabi carved in cuneiform (Fig. 1) and the Egyptian papyrus medical books written in hieroglyphic alphabets (Fig. 2) left us the earliest records of Western traditional medicine while the oracle inscriptions of the Shang Dynasty inscribed on tortoiseshells or animal bones that discovered in Yin remains (Fig. 3) demonstrated the initial knowledge of the ancient Chinese people about medicine. (There was no concept of TCM at that time.) Probably from that time on, traditional medicine, which is essentially empirical medicine, appeared successively but almost coincidentally in the East and West in Mesopotamia, the Nile valley, the Yellow River basin, and other regions that held the birth of human civilization, heralding the early dawn of human medicine.

Since ancient people had poor capacity of recognizing and reforming the nature, primitive religion almost invariably became an important source of human knowledge including medicine in the world. At this point, early medicine experienced a history of coexistence with religion in its huge

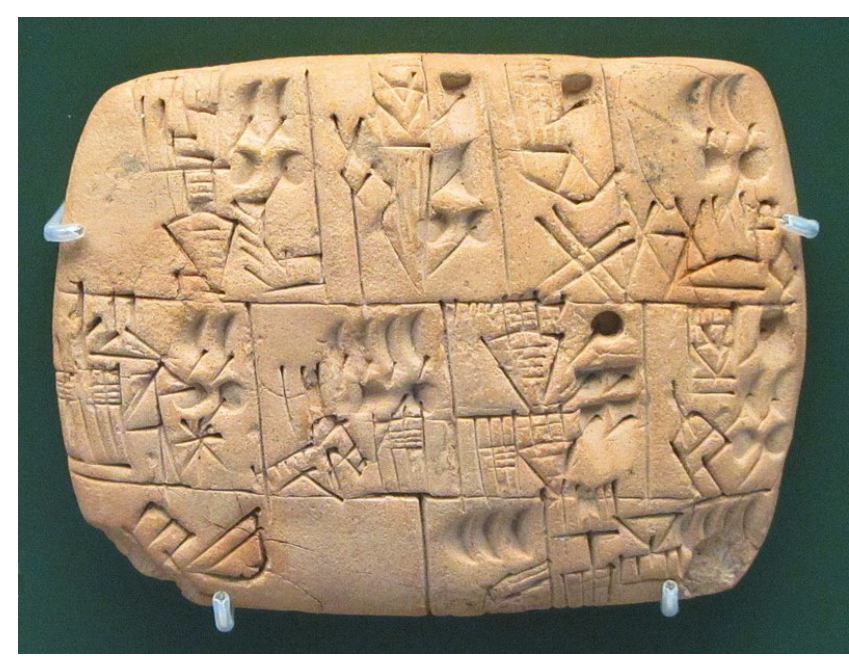

Figure 1. The manuscript carved in cuneiform can be dated back to about 3000 B.C., which was discovered in Southern Iraq.

Source: https://en.wikipedia.org/wiki/Cuneiform\#/media/File:Early_writing_ tablet_recording_the_allocation_of_beer.jpg.

system. As it is said in the Chapter of Changing Essence and $Q i$ in Plain Questions in Inner Canon of Yellow Emperor, people believed that diseases could be treated by supplication to change body's state of Essence and $Q i$ in ancient times. ${ }^{10}$ The prevalence of supplication in ancient Chinese is not much different from that in ancient Greeks who visited the God of medicine Asclepius and his temples to receive dream therapy. Witch doctor is the earliest doctor while witchcraft is the earliest medical skill, and without exception, "the combination of medicine and witch" is the early characteristic and necessary stage of Chinese and Western traditional medicine.

The ancient people who gradually stepped into the civilized era were also trying to trace the origin of medicine, just

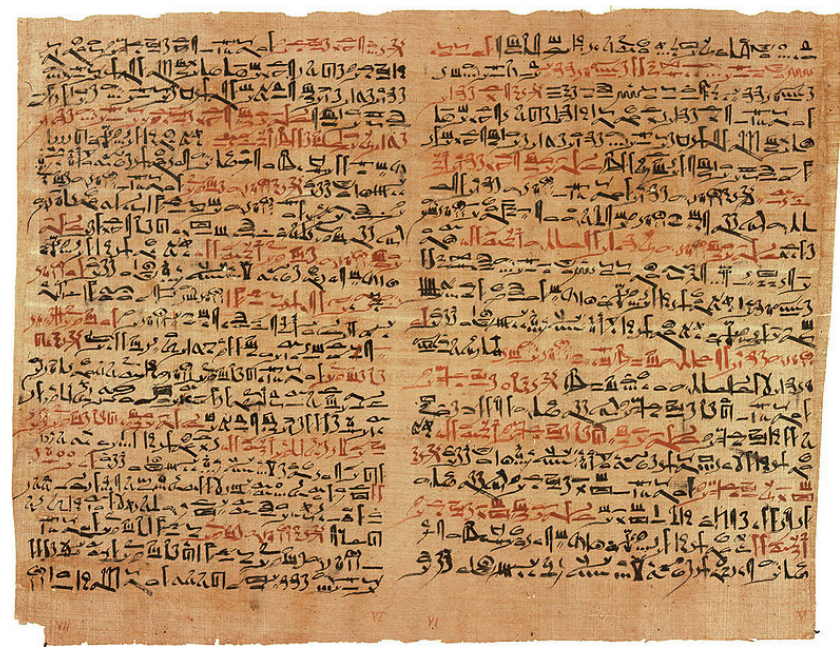

Figure 2. The Edwin Smith Papyrus.

Source: https://en.wikipedia.org/wiki/Edwin_Smith_Papyrus\#/media/File: Edwin_Smith_Papyrus_v2.jpg. 


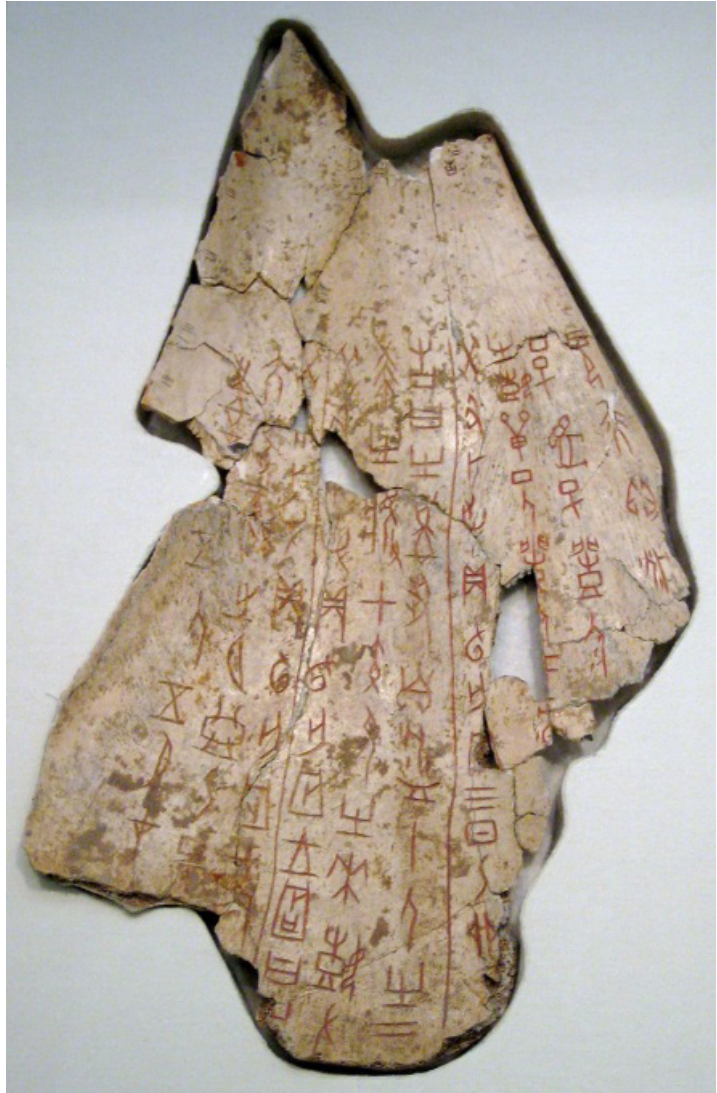

Figure 3. The oracle inscriptions of the shang dynasty.

Source: https://en.wikipedia.org/wiki/Oracle\#/media/File:Shang_dynasty_ inscribed_scapula.jpg.

like what we do today. For example, there have been related legends and views in China such as "Shennong tasted hundreds of herbs and found more than a hundred of remedies, and thus medicine originated from his practice", "Fuxi made needles for acupuncture" and so on. In fact, as far as how medicine originated is concerned, we do not seem to know more than the ancient people did because of the long history, insufficient cultural relics and other reasons. After all, the fictional images of the sages, such as Shennong and Fuxi, represented the initial exploration of Chinese people in medicine, and are thus regarded as the origin of TCM. Then the primitive medical knowledge and clinical experience continued to accumulate and the medical knowledge system was gradually constructed under the guidance, influence and stimulation of regional cultures, ancient philosophy and religious beliefs. Medicine began to enter the process of independent development, and medicine consisting of original, simple, and scattered medical knowledge and experience gradually evolved into a medical system characterized by logical relationships, systematic medical theories, and rich practical experience.

However, influenced by different group beliefs, classical philosophy, regional culture and other elements, Eastern and Western traditional medicine are developing differently. For example, ancient Greek medicine takes humoral temperament theory as its basic theoretical framework while TCM chooses the theory of Yin and Yang and Five Key Elements (Fig. 4). In general, however, it is still in the scope of empirical medicine, and its essence is the individualized embodiment of medical thinking and practice of different philosophical backgrounds, cultural backgrounds, religious ideas, and cognitive methods in both China and the West. It can be understood that different ethnic cultures endow their traditional medicines with several superficial structural features, but they share the same basic core. For example, they both pay attention to the idea of the harmony between human and nature; they both focus on the balance of homeostasis in which the West stresses the balance of body fluid while China stresses the balance of Yin and Yang; they both
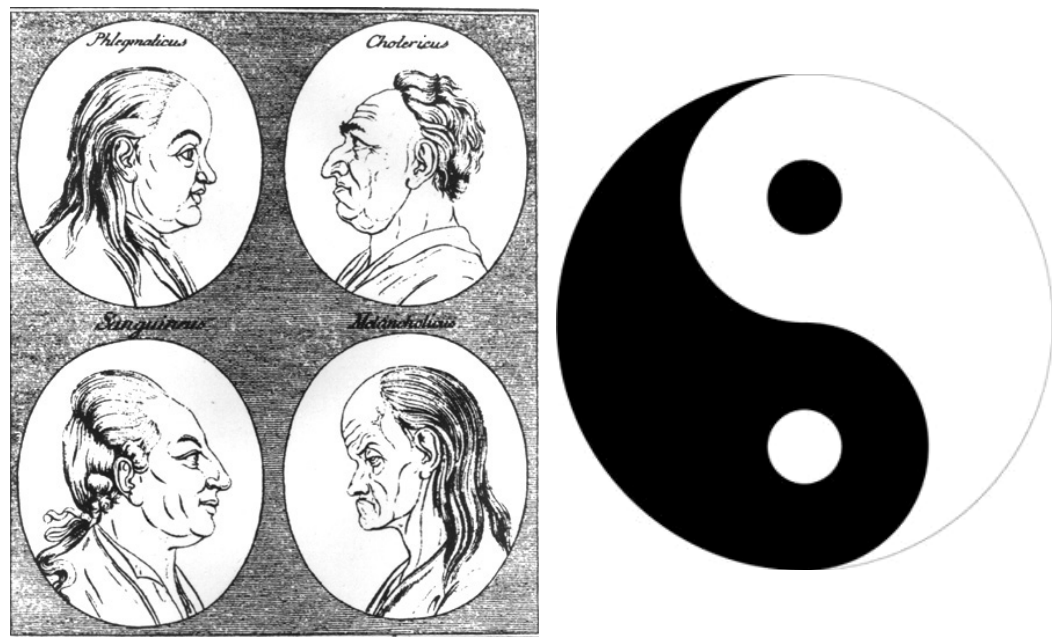

Figure 4. The theory of humoral temperament (left) and that of Yin and Yang (right). Source: https://en.wikipedia.org/wiki/Four_temperaments\#/media/File:Lavater1792.jpg. 
generally adopt the methods of "filling up the deficiency and relieving the excess", "treating coldness with heat and treating heat with cold", etc., and eventually maintain the body in a balanced status. Another example is the vein. As it is the channel of some important information of human life and blood flow, there undoubtedly contains some potential information about the conditions or the symptoms and physical signs of the human body; on the other hand, as the pulse is usually located at the superficial and obvious parts of the human body, it is undoubtedly that pulse-taking is a medical behavior that is close to human instinct. It is a simple and practical way to examine the condition of the human body, and it is also a common choice made by different classical medicines. It is neither unique to TCM nor to the East. To a large extent, the differences between them are reflected in different ways of philosophical thinking, religious customs and cultural backgrounds, and the differentiated cognition and specific practice of physiological phenomena and medical diagnosis methods, such as the human meridian blood vessels and pulse-taking. For example, in Western countries, it is mainly to touch the heart pulse of the whole body without going beyond the relationship between the pulse and the heart while in China, it is usually to touch the numerous pulses reflecting the information of different parts of the human body. This is the philosophical foundation and cultural differences behind Chinese and Western sphygmology. However, the internal differences of TCM (such as the differences between TCM of different ethnic groups) are not reflected in the philosophical theoretical basis and methodology of pulse diagnosis, but in some specific concepts and clinical implications of pulse diagnosis, which are the internal disputes within the same sphygmology system. Therefore, in general, the divergence is the necessity of the development of Eastern and Western traditional medicine while the convergence is the requirement of the essence of Eastern and Western traditional medicine.

The first peak of the development of traditional medicine appeared in the Axial Age proposed by the German thinker Karl Jaspers. In his book The Origin and Goal of History, he called the breakthroughs of human culture simultaneously occurring in regions like China, the West and India around 500 B.C. as the "Axial Age". Both Western traditional medicine represented by Hippocrates and his writings and CTM represented by Inner Canon of the Yellow Emperor originated at that time. From the perspectives of the development and content of medicine at that time, both Chinese and Western traditional medicine showed the characteristics of abandoning the theological witchcraft, penetrating philosophy, exploring the characteristics of medicine itself and constructing the medical theory system, and for the first time in the history of medical civilization, the unprecedented prosperity of Chinese and Western medicine reflected mutually.
In the development of Axial Age, Chinese and Western traditional medicine together completed the basic construction of medicine, respectively, and most of the medicine appearing subsequently continued their new journey under the guidance of The "Hippocratic" Corpus (Fig. 5) and Inner Canon of the Yellow Emperor, such as the Roman medical achievements represented by Galenus (Fig. 6) and the development of TCM represented by Zhang Zhongjing (Fig. 7). The former inherited the tradition of Hippocratic medicine and made great achievements in the primitive acrasiology, anatomy and physiology, which improved the medical system of Western traditional medicine focusing on anatomy, clinical observation and demonstration, while the latter laid the foundation of Syndrome Differentiation and Treatment (Bian Zheng Lun Zhi) as well asTheories-Diagnostics-Prescriptions-Drugs (Li Fa Fang Yao) and promoted the integration of theory and clinical practice of TCM. With the emergence of the four TCM classics, Inner Canon of Yellow Emperor (Huang Di Nei Jing), Yellow Emperor's Canon of 81 Difficult Issues (Nan Jing), Shennong's Classic of Materia Medica (Shen Nong Ben Cao Jing) and Treatise on Febrile and Miscellaneous Diseases (Shang Han Za Bing Lun), TCM also initially completed its own systematic construction during this period. It can be regarded as the continuation of

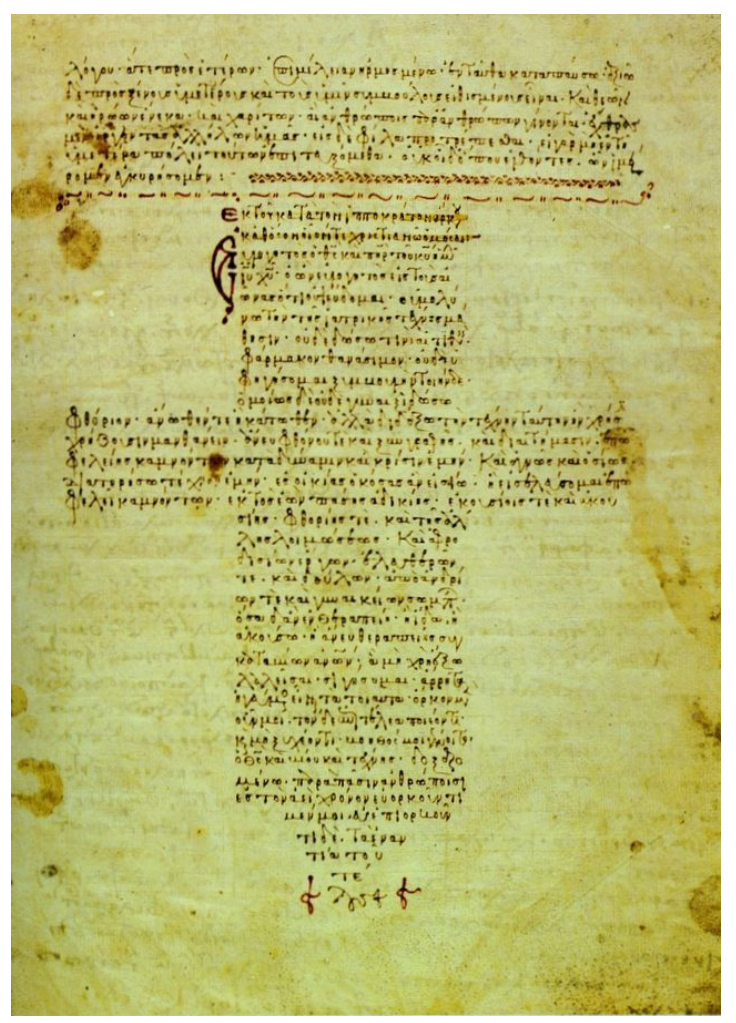

Figure 5. The manuscript of hippocratic oath in the 12th century. Source: https://en.wikipedia.org/wiki/Hippocrates\#/media/File:HippocraticOath.jpg. 


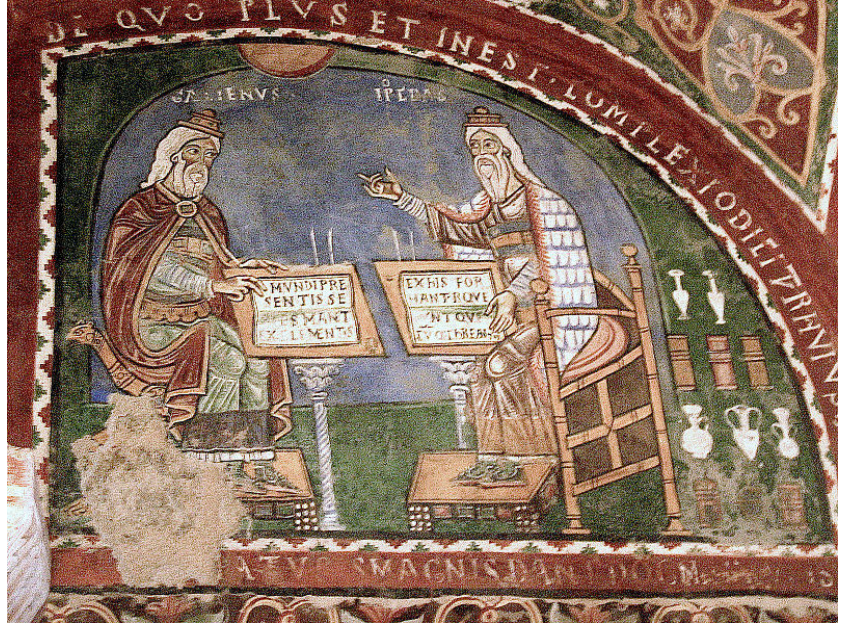

Figure 6. The portrait of Hippocrates and Galenus created by an Italian artist in the 12th century.

Source: https://en.wikipedia.org/wiki/Hippocrates\#/media/File:Galenoghippokrates.jpg

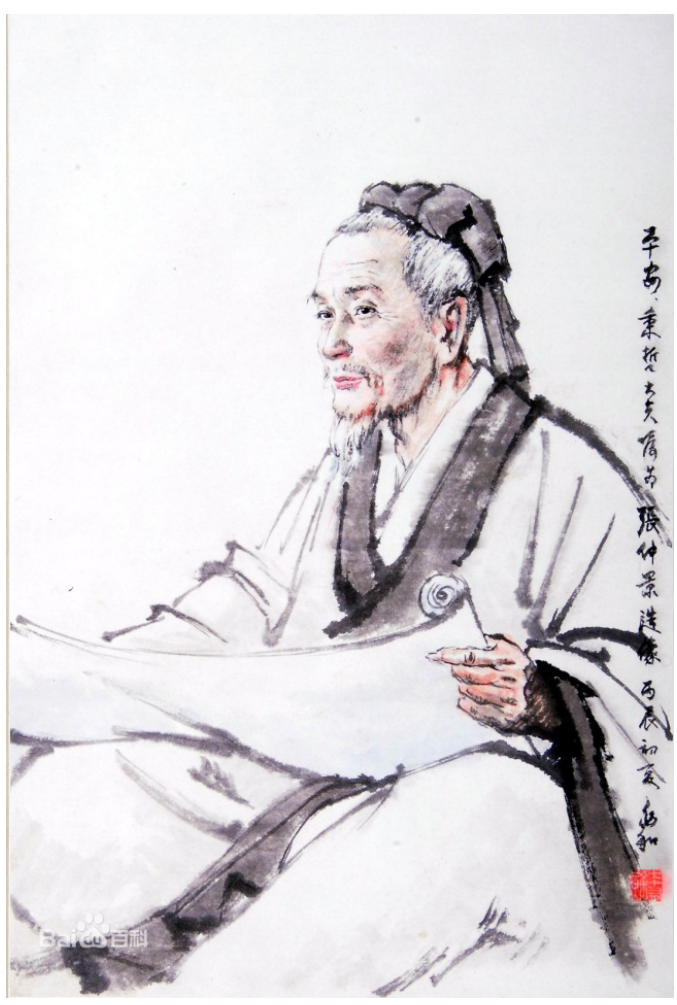

Figure 7. The portrait of Zhang Zhongjing

Source: https://baike.baidu.com/pic//66566/0/f2deb48f8c5494ee9451190f20 f5e0fe 99257 eb0 $? \mathrm{fr}=$ lemma \&ct=single\#aid $=0 \&$ pic $=7 \mathrm{e} 3 \mathrm{e} 6709 \mathrm{c} 93 \mathrm{~d} 70 \mathrm{cf}$ 48 fadcd 8 f1dcd100bba12be6.

the Axial Age and the second peak in the history of both TCM and Western traditional medicine.

After that, Western traditional medicine, especially the ancient Greek-Roman medicine represented by Hippocrates and Galenus, continued to develop in the Arab region, with

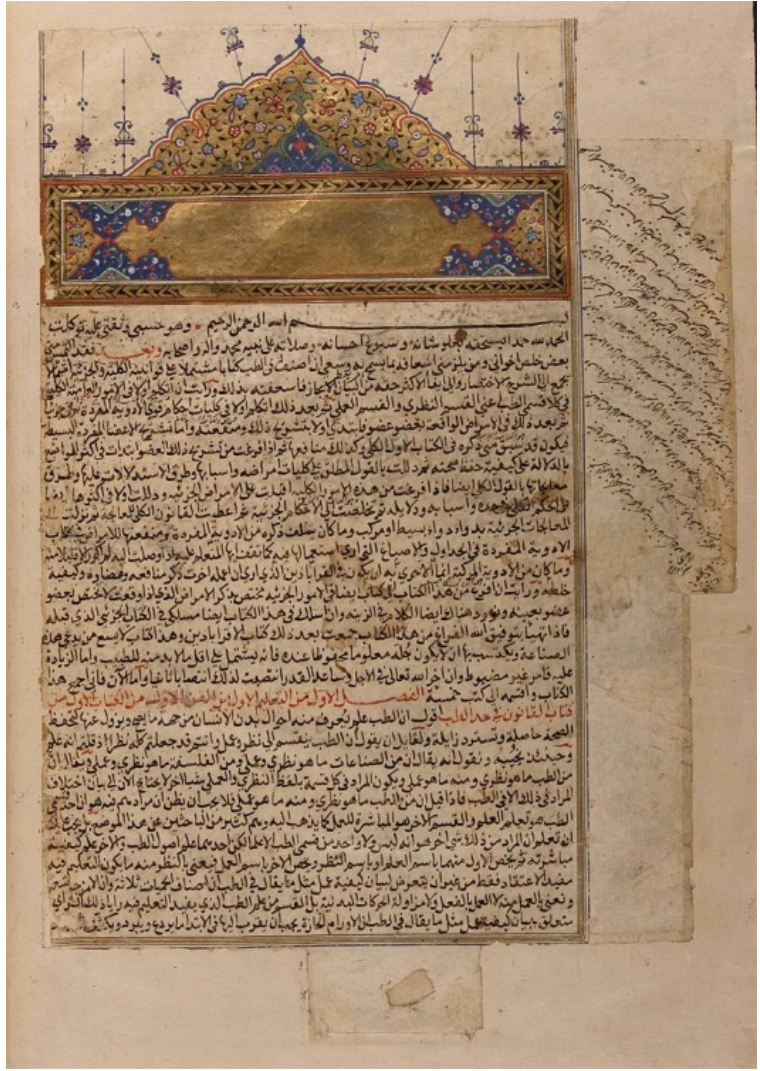

Figure 8. The manuscript of Canon of Medicine written by Avicenna which is preserved in Yale University.

Source: https://en.wikipedia.org/wiki/Avicenna\#/media/File:Avicenna_canon_ 1597.jpg.

the emergence of Avicenna and his influential medical work Canon of Medicine (Fig. 8). But since then, Western medicine obviously changed from prosperity to decline and entered the stagnation of medieval medicine. On the other hand, TCM, under the continuous nourishment of Chinese civilization and culture, continued to maintain the steady development, and reached a peak in the later period, especially in Song, Jin and Yuan Dynasties. Although the development of TCM was not as stagnant as that of Western medicine in the same period, it also showed signs of mere inheritance rather than further innovation, leading to the significantly slower development and achievements than that of the earlier period.

Over a long period of time, influenced by the changes in the Chinese territory, the thinking of Da Yi Tong that refers to political, economic, and cultural integration, the emergence of communications brought about by the social development as well as the inclusiveness of Chinese civilization, the communication among various ethnic groups in China have become increasingly frequent, and ethnic traditional medicines have also been increasingly developed. Actually, most of these ethnic traditional medicines are neither indigenous 
nor introduced, but formed on the basis of Chinese traditional cultures, basic medical knowledge, and clinical practice of ethnic minorities. In brief, they are the combination of TCM and the excellent traditional cultures of various ethnic minorities. For example, Uyghur Medicine and Hui Medicine have been deeply rooted in TCM, and they have also absorbed the essence of ancient Persian medicine, ancient Indian medicine, and Arab medicine. Thus, traditional medicines of different ethnicities in China contribute to the development of CTM ( $\mathrm{Da}$ Zhong Yi).

By comparing the history of TCM and Western traditional medicine, we have found that although there are differences in origination, development and level, one is not superior to the other. On one hand, rooted in the flourishing Chinese civilization, TCM has kept steadily developing, which tends to be more conservative; on the other hand, influenced by the fading Babylonian civilization, Egyptian civilization, Greek civilization, and Roman civilization, Western traditional medicine did not develop continuously, which are likely to be more flexible and malleable, and thus clarifying, to some extent, the reasons why Western medicine has changed radically in the overall transformation of Western society.

The important turning point of medicine is undoubtedly the birth of modern medicine. The Renaissance and the Scientific and Technological Revolution nourished modern medicine, leading to radical changes in Western traditional medicine and renewed development of medicine in the West and even the whole world. Since then, modern medicine has completely replaced Western traditional medicine and has gradually become predominant in the West, which was once considered the only medical science.

"When studying the development of human history, we can conclude that the progress of civilization relies on broadening the horizons ${ }^{11}$ " (Lv Simian, The General History of China). With the enrichment of the knowledge of geography and the discovery of new waterways, the world has become increasingly integrated, and all the disciplines including medicine begin to communicate unprecedentedly.

With the openness to the world and the eastward spread of Western culture and science after the Opium War, modern medical concepts and technologies have flooded into China and greatly impacted TCM. Consistent with the national reforms and the desire of saving the nation from subjugation and struggling to survive, the medical profession also initiated reforms and salvation activities. These activities eventually led to the consequences that the dominance of TCM in Chinese medicine waned, and the confluence and the subsequent integration of TCM and modern medicine emerged and flourished. Different from the way that modern medicine directly replaces traditional medicine in the West, the coexistence and integration of TCM and modern medicine is adopted in China. It vigorously develops modern medicine and simultaneously retains traditional medicine with its characteristics and advantages. The change of the relationship between TCM and modern medicine in China from confrontation to integration not only indicates that modern medicine is advanced in some aspects and has an impact on traditional medicine, but also that TCM is a relatively advanced traditional medicine and has been widely used by a wide range of people with good therapeutic effects. ${ }^{12}$ The inheritance, innovation, and development of TCM can be attributed to the diversity and inclusiveness of Chinese civilization, which can also explain why Chinese civilization has been inherited for 5000 years. Now, integrative medicine is a significant driving force of medical development, and has become one of the features in Chinese medicine.

\section{Medicine at Present}

Stimulated by the Industrial Revolution and the Scientific and Technological Revolution, modern medicine has achieved rapid development in only a few hundred years. Human anatomy represented by Vesalius in the 16th century, human physiology represented by Harvey in the 17th century, organ pathology represented by Morgagni in the 18th century, cytopathology represented by Virchow and microbiology represented by Pasteur in the 19th century, clinical medicine prospering in the 20th century, and other disciplines such as medical imaging, immunology, genetics, preventive medicine, biomedicine, and molecular medicine have made great achievements. The continuous development has laid the foundation of brilliant modern medicine. By the middle of the 20th century, infectious diseases that had previously threatened human life were basically controlled, and the average life expectancy reached an unprecedentedly high level. At the same time, the medical thoughts guided by reductionism replaced that guided by pathophysiology in the humoral temperament theory. With the help of science and technology, modern medicine gradually evolved from macroscopic to microscopic level. Advanced medical concepts and therapeutic methods such as evidence-based medicine, epidemiology, precision medicine, targeted therapy, immunotherapy, and gene editing are emerging. Modern medicine, which is different from traditional medicine, has created miracles in the development of medicine in just a few centuries.

Although modern medicine has been leaping forward, history and practice have increasingly shown that advanced modern medicine is not omnipotent. Medicine is facing new problems and challenges at present. For example, some infectious diseases that have been conquered or even disappeared, such as malaria and tuberculosis, seem to come back; due to the presence of drug resistance, there have been 
increasing questions on antibiotics, with the fact that Ebola, Zika, Middle East Respiratory Syndrome (MERS), and other super viruses have posed new threats to human; research and treatment on AIDS and cancer have not yet made major breakthroughs; in the treatment of common diseases and chronic diseases, modern medicine has poorly performed, and the pathogenesis of some neurodegenerative diseases like Alzheimer's disease has not been fully explained. In addition, diagnostic techniques in modern medicine are getting more and more advanced, but the level of diagnosis and satisfaction of doctors and patients have not been improved simultaneously. Many problems in the field of medical humanities remain to be solved with the development of modern medicine.

Of course, not only modern medicine but also traditional medicine has the responsibility and obligation to solve the problems mentioned above. They are not caused by modern medicine, and there is no doubt that modern medicine has greatly advanced the progress of medical development and improved the human health. These problems remain to be solved by modern medicine or medicine.

There are also many problems in the development of traditional medicine nowadays. From the perspective of traditional medicine in the world, Western traditional medicine represented by Hippocrates, once popular in European and Arab countries, has waned currently; similarly, Ayurvedic medicine has not been well inherited in India, and only skills like yoga are prevalent in the world. In the ancient civilizations and their unique traditional medicines, only Chinese civilization along with TCM has been well inherited and innovated theoretically and practically. Traditional medicine was once faced with oppositions in Western countries because of the development of modern medicine, and now the so-called complementary/alternative medicine begins to attract the attentions. Does it indicate, to some extent, the revival of traditional medicine worldwide and the establishment of a new medical system, or the attempt of modern medicine to seek help and support from traditional medicine? These are important medical problems worth exploring and solving. Likewise, it should be noted that there are some arguments in TCM. First, there are still inaccurate concepts and understandings that might affect its positive development, such as the definition of "Chinese medicine", "Western medicine", "CTM", "traditional medicine", "ethnic traditional medicine", "Han Medicine", and how to correctly understand and apply traditional medicine, which have caused the problems ranging from the sense of Chinese national identity to the diagnosis and treatment of every patient. ${ }^{7}$ Second, the concentrated and conservative development of traditional medicines of different ethnicities, such as Tibetan Medicine, Mongolian Medicine, Uyghur Medicine, and Dai Medicine, has hindered their communication and interaction, which could in turn explain their stagnation and monotonicity. Solving such problems can not only promote the development of TCM, but also improve its competitiveness and influence, and simultaneously add to the sense of the community of all ethnicities in China.

The attribution and categorization of different ethnic traditional medicines in CTM (Da Zhong Yi) is what cannot be neglected in the process of inheritance and innovation, and we take Uyghur Medicine for example.

In general, philosophical concepts are often added into the ontology of traditional medicine. Specifically, medical ontology focuses on science and technology while philosophical concepts tend to reflect the worldview and methodology behind traditional medicine. Traditional medicine is basically composed of five elements: clinical experience, ancient philosophy, regional culture, religious belief, and primitive knowledge of medicine. Primitive knowledge of medicine and clinical experience are related to medical techniques, and its continuous absorption of knowledge from other medicines will be beneficial to traditional medicine. However, changes in ancient philosophy, regional culture or religious belief, i.e., philosophical concepts and theories, will undoubtedly lead to the changes of traditional medicine itself, which will involve the problems of its attribution and even categorization. Therefore, the attribution and categorization of different ethnic traditional medicines is not only a comparison of their techniques, but also their philosophical basis and methodology underlying, and the latter are often the key factors.

Uyghur Medicine was originated in Western China (now Xinjiang). Due to the fact that Xinjiang is located in the border of China, Uyghur Medicine has communicated more frequently with foreign medical cultures, but they still have some interactions with other domestic traditional medicines in the process of origination and inheritance. The theories of Uyghur Medicine originated from the drug usage and clinical practice in Xinjiang and later absorbed the essence of other domestic traditional medicines, which is the combination of TCM and the excellent Uyghur cultures. From the aspect of Syndrome Differentiation and Treatment (Bian Zheng Lun Zhi) in Uyghur Medicine, Uyghur doctors, like TCM (Han Medicine) practitioners, always focus on patients or those who are prone to illness, with the diagnostic methods including inspection, auscultation and olfaction, inquiry, pulse-taking and palpation, drawing a conclusion similar to Syndrome (Zheng) in TCM (Han Medicine). With the development of modern life sciences, its evaluation of efficacy has gradually become consistent with integrative medicine, i.e., the evaluation with both TCM (Han Medicine) and modern medicine. Although the explanations of pathogenesis and the methods of treatment vary in ethnic traditional medicines, the similarities outweigh the differences. 
Historically, genuine crude drugs from Xinjiang were continuously introduced into other regions in Mainland China, which were further absorbed by TCM (Han Medicine) and named traditional Chinese herbs (Zhong Yao). Later, Uyghur Medicine learned the principles of compound prescriptions and the rules of naming, some of which, according to our research, were consistent with that of TCM (Han Medicine), especially the prescriptions in modern times. These prescriptions are usually named after "principal efficacy plus dosage form", "diseases to be treated", "principal herbs in the prescription", "dosage plus dosage form" or "principal herbs in the prescription plus dosage form". At the same time, most of the instructions for proprietary Uyghur Medicine are similar with the statements in TCM (Han Medicine) that instead of some specific concepts and names in Uyghur Medicine, their principal efficacy and indication can be clearly explained by the terms in TCM (Han Medicine). In clinical practice, the principles of proprietary Uyghur Medicine are usually explained with the theories of TCM (Han Medicine), while unique terms from Uyghur Medicine are more likely to be used for the purpose of academic exchanges or the conservation of Uyghur Medicine.

Therefore, Uyghur Medicine can be regarded as the combination of primitive clinical experience and medication, which is profoundly influenced by the cultures of TCM (Han Medicine). Although ethnic traditional medicines have their own characteristics, actually most of them have evolved from the cultures of TCM (Han Medicine), which are the combination of TCM and the excellent traditional cultures of various ethnic minorities. They have gradually formed their own theoretical systems in the long-term development, but they are still commensurate with TCM (Han Medicine) in terms of origination, some theories, diagnosis, and treatment. Therefore, the problems of the relationship of different traditional medicines, and furthermore, their attribution and categorization within the system of CTM, are worthy of our considerations and investigations.

\section{Medicine in the Future}

Modern medicine has limitations in some fields. On one hand, it inspires contemporary medical scientists and clinical experts to continuously deepen medical research, improve medical technology, overcome medical problems, and look forward to the brilliant future of medicine. On the other hand, it also urges people to view modern medicine as well as traditional medicine more rationally and dialectically. Nowadays, besides modern medicine, the so-called complementary/alternative medicine is more and more popular in European countries, America, and other countries around the world. Meditation therapy, hypnotherapy, homeopathy, massage therapy, aromatherapy, vitamin therapy, and acupuncture, traditional Chinese herbs, Qigong, Tai Chi from China as well as yoga from India are all included in the category of complementary/alternative medicine. In China, people have the same idea. While relying on modern medicine, people still believe in CTM, including Han Medicine, Tibetan Medicine, Mongolian Medicine, Uyghur Medicine, Dai Medicine, and so on. According to different conditions of different diseases, modern medicine, traditional medicine, or integrative medicine that combines the advantages of them will be selected, which has become the consensus of both doctors and patients.

We put forward the concept of Five Key Elements (Wu Yao $\mathrm{Su}$ ) and Trichotomy (San Fen Fa) to correctly and rationally understand traditional medicine, modern medicine, and their coexistence. Five Key Elements (Wu Yao Su) refer to clinical experience, ancient philosophy, regional culture, religious belief, and primitive knowledge of medicine, which integratively frame any traditional medicine. Trichotomy (San Fen $\mathrm{Fa}$ ) refers to the fact that the similarities outweigh the differences in ethnic traditional medicines, and their basic structure can be divided into the following three parts: the part that is unconsciously ahead of modern medicine, the part that has already reached consensus with modern medicine, and the part that needs to be reconsidered or abandoned. Traditional medicine and modern medicine, with their own characteristics and merits, belong to different medical systems, and to some extent, traditional medicine cannot be replaced by modern medicine and vice versa. For example, CTM is now the most mature traditional medicine that absorbs and integrates the essence of experience-based medicine, and its comprehensive system that is filled with practical medical technology and medical experience gradually recognized by modern medicine indicates the direction and advancement of medicine in the future. Therefore, it is sensible that we take advantage of both traditional medicine and modern medicine harmoniously for the sake of conquering diseases and maintaining health. Of course, it should be admitted that essence and dregs coexist in traditional medicine throughout its development, so traditional medicine keeps its evolution and improvement in continuous sublation and rejection.

The development of history and times has increasingly proved that traditional medicine and modern medicine, with their own characteristics and merits, belong to different medical systems, and it is the right way to seek similarities while reserving differences. In the future, medical integration will be the general trend. ${ }^{5}$ Although the development of modern medicine skyrocketed in a history of about 400 years, it is no more than a short period of time in the development of medicine. Long before the birth of modern medicine, medicine tends to be more conservative due to geographical distances and inconvenient transportation. At that time, there was no distinction between traditional medicine and modern 
medicine, Chinese medicine and Western medicine, and between ethnic traditional medicines nowadays such as Tibetan Medicine, Mongolian Medicine, Uyghur Medicine or Dai Medicine, etc. People only had the concepts of life and death, health and disease, doctors and patients, and medical practice constituted by these elements. From the perspective of concept and connotation, it might be the essence and origin of medicine. In fact, medicine is a kind of science and practice that aims to relieve pain, prevent and treat diseases and improve health. It might be the future direction of medical research to investigate into the universality and essence of medicine.

What should we do about the integration and development of medicine in the future? The coexistence of traditional medicine and modern medicine nowadays as well as the situations, problems and challenges of medicine around the world will guide the continuous development of medicine. Although modern medicine has been recognized as our common medical civilization and science, traditional medicine might help to take it further. Only through the combination of traditional medicine and modern medicine, we can create a more brilliant medical civilization. ${ }^{5}$ Based on what we mentioned above, we put forward the concept of Three-dimensional Integration (San Rong He): First, the integration of traditional medicines among different ethnicities in China to establish a new system of TCM based on the community of all ethnicities in China; second, the integration of traditional medicines from around the world to establish a new system of global traditional medicine based on the community of a shared future for mankind; third, the integration of traditional medicine and modern medicine to explore the essence as well as enrich the connotation of traditional medicine with the help of the techniques, theories and methods of modern science, which will simultaneously promote the development of modern medicine. Those three parts are not sequential, but parallel.

Admittedly, we should be aware that although it is important to inherit and innovate traditional medicine, modern medicine, with its inseparable relationship with modern science and technology, has been recognized as our common medical civilization and science, which is predominant in the field of medicine. The rapid development of science and technology, such as the emergence of disciplines like quantum mechanics and artificial intelligence, will definitely give impetus to the development of medicine.

What will medicine be like in the future? On one hand, we try to develop modern medicine with the help of modern science and technology, and it is a process of constant revision and improvement, gradually approaching absolute truth. On the other hand, we should emphasize the importance and indispensability of traditional medicine simultaneously, and it is also a good opportunity to extract the essence of traditional medicine so that traditional medicine will shine brightly in contemporary medical civilization. Take TCM (Zhong Yi) for example, we should focus on the inheritance and innovation of its unique quality, especially the original thinking such as the holistic view based on the Correspondence between Man and Nature (Tian Ren He Yi), the individualized therapy based on Syndrome Differentiation and Treatment (Bian Zheng Lun Zhi), the clinical experience based on TheoriesDiagnostics-Prescriptions-Drugs (Li Fa Fang Yao) and the theory of health preservation based on the Prophylactic Treatment of Diseases (Zhi Wei Bing). When adhering to the essence of TCM (Zhong Yi), we should also be open to modern medicine and traditional medicine for the purpose of their further development and improvement. Consequently, traditional medicine and modern medicine should be guided and strengthened by integration, and thus continuously amplifying the characteristics and advantages, enriching the connotation of TCM together with promoting the development of modern medicine, which will be a powerful propellant to the establishment of a brand new system of medicine that harmoniously integrates traditional medicine and modern medicine. ${ }^{5}$ We firmly believe that in the future, every medical system will keep its uniqueness, and simultaneously the advantages of other medical systems will be appreciated in great esteem. If medicine represents itself with diversity and integrity just like a grand and harmonious symphony, the whole world will be blessed with harmony and unity. Ultimately, medicine is the science of protecting and promoting human health, and we should bear in mind the essence and goal of medicine when we try to go further, never forgetting why we started.

\section{Acknowledgments}

This work was supported by the grant from China Postdoctoral Science Foundation (No. 2017M621369).

\section{Conflict of Interest}

The authors declare no conflict of interest.

\section{References}

1. $\mathrm{Ci}$ Hai, An unabridged and comprehensive dictionary of Chinese. Shanghai: Shanghai Cishu Publishing House, 1979:383.

2. The Contemporary Chinese Dictionary (Version VI). Beijing: The Commercial Press, 2014:1532. 
3. https://en.wikipedia.org/wiki/Medicine, https://www.who.int/ topics/traditional_medicine/zh/.

4. WHO Traditional Medicine Strategy: 2014-2023, World Health Organization 2013:15.

5. Dong JC. The evolution of traditional Chinese medicine as a disciplinary concept and its essence throughout history. People's Forum: Acad Front 2018;9:62-68.

6. Dong JC. Philosophical thinking of Chinese traditional medicine (CTM). People's Daily 17 October 2014.

7. Dong JC. Philosophical implications of Chinese traditional medicine (CTM). People's Forum: Acad Front 2014;18:84-94.

8. "Talks with musicians". The History of Integrative Medicine. Beijing: China Science and Technology Press, 2010:3.
9. Cheng ZF, Zhen C. A History of Medicine (Volume One). Nanjing: Yilin Press, 2014:9.

10. Guo AC. Inner Canon of Yellow Emperor - Plain Questions (Huang Di Nei Jing · Su Wen) (Volume One). Beijing: China Press of Traditional Chinese Medicine, 2016:83.

11. Lv SM. The General History of China. Shanghai: Shanghai Classics Publishing House, 2009:75.

12. Liu WX, Lu LW, Ma $\mathrm{C}$ et al. The evolution of traditional Chinese medicine as a disciplinary concept and its essence throughout history. Tradit Med Mod Med, 2018;1(3):171-180. 